\title{
ORT. I 2 - Evaluation of the antitumor effect of Natural Killer cells expressing Chimeric Antigen Receptors (CAR) against murine melanoma cells
}

\author{
Mariana Saldanha Viegas Duarte ${ }^{1 *}$; Luiza de Macedo Abdo'; Luciana Rodrigues Carvalho \\ Barros $^{1}$; Martín Hernán Bonamino ${ }^{1}$.
}

1INCa.

Introduction: Natural Killer (NK) cells are an important component of the innate immune system, having a classic role in providing anti-tumoral and antiviral immunity. These cells are potential effectors in allogeneic cancer immunotherapy, mediating antitumor effects without inducing potentially lethal alorreativity, such as graft versus host disease (GVHD). The lack of specificity and targeting of these cells is a limiting factor, which can be solved by inducing the expression of chimeric antigen receptors (CARs). CARs consist of an extracellular domain based on the immunoglobulin variable region ( $\mathrm{scFv}$ ) and an intracellular signaling domain based on the CD3 complex zeta chain, which may or may not be complemented with costimulatory sequences. Melanoma is a cancer with a high level of lethality, mainly in its metastatic form, requiring new therapeutic approaches aimed at increasing the survival of patients.

Objective: Establish a cancer immunotherapy model using NK cells loaded with CARs via a trogocitosis-mediated CAR transfer.

Methodology: NOD SCID mice were engrafted subcutaneously with the murine melanoma B16F10 cell line. Tumor growth kinetics of the parental B16F10 and its derivative CD19+ and/or CD20+ cells were determined. Human NK cells received CARs through trogocitosis, a mechanism of molecule transfer by cell to cell contact. NK cells were co-incubated with the K562 cells expressing the 19BBz CAR (anti-CD19), the 20BBz CAR (anti-CD20) or both constructs. The cytotoxic potential of NK cells expressing CAR $19 \mathrm{BBz}$ and/or $20 \mathrm{BBz}$ against wild-type or modified B16F10 murine melanoma cells for expression of human CD19 and/or CD20 proteins was evaluated in vitro by cell lysis assays.

Results: In the model the amount of $1 \times 10^{5}$ cells was established for the parental B16F10, $\mathrm{CD} 19+\mathrm{B} 16 \mathrm{~F} 10, \mathrm{CD} 19+\mathrm{CD} 20+\mathrm{B} 16 \mathrm{~F} 10$ and $5 \times 10^{5}$ cells for the CD20+ B16F10 lineage in male mice. CAR trogocysotis showed high efficiency, ranging from $67.9 \%$ to $99.1 \%$ CAR + NK cell population. Results obtained in the in vitro cytotoxicity assays for analysis of the lytic potential of human NK cells against B16F10 murine melanoma lines, expressing as target antigen the human CD19 and CD20 proteins, resulted in a low specific lysis. The lytic potential of $19 \mathrm{BBz}$ $20 \mathrm{BBz}$ NK cells against the CD19 + CD20 B16F10 line showed greater cytotoxic potential, suggesting two different target antigens can promote efficient lysis.

Conclusion: Trogocytosis showed to be an efficient method for loading NK cells with CAR molecules. The melanoma model derived from the parental B16F10 cell lines and their modifications in immunodeficient mice was efficiently established for the standardization of tumor growth. This model is an important tool for future evaluation of the anti-tumor potential of CAR+ NK and, potentially, T cells.

Keywords: Natural Killer (NK); CAR; cancer immunotherapy 